\title{
The ring statistics - how to separate E- and B-modes of cosmic shear correlation functions on a finite interval
}

\author{
P. Schneider and M. Kilbinger
}

\author{
Argelander-Institut für Astronomie ${ }^{\star}$, Universität Bonn, Auf dem Hügel 71, 53121 Bonn, Germany \\ e-mail: [peter;kilbinge] @astro.uni-bonn.de
}

Received 2 May 2006 / Accepted 29 September 2006

\begin{abstract}
Aims. Cosmic shear, the distortion of images of distant sources by the tidal gravitational field of the large-scale matter distribution in the Universe, is one of the most powerful cosmological probes. The measured shear field may be due not only to the gravitational lensing effect, but may contain systematic effects from the measurement process or intrinsic alignment of galaxy shapes. One of the main probes for these systematics is the division of the shear field into E- and B-mode shear, where lensing only produces the former. As shown in a recent note, all currently used E-/B-mode separation methods for the shear correlation functions $\xi_{ \pm}$require them to be measured to arbitrarily small and/or large separations which is of course not feasible in practice.

Methods. We derive second-order shear statistics which provide a clean separation into E- and B-modes from measurements of $\xi_{ \pm}(\vartheta)$ over a finite interval only. We call these new statistics the circle and ring statistics, respectively; the latter is obtained by an integral over the former. The mathematical properties of these new shear statistics are obtained, as well as specific expressions for applying them to observed data.

Results. It is shown that an E-/B-mode separation can be performed on measurements of $\xi_{ \pm}$over a finite interval in angular separation, using the ring statistics. We furthermore generalize this result to derive the most general class of second-order shear statistics which provide a separation of E- and B-mode shear on a given angular interval $\vartheta_{\min } \leq \vartheta \leq \vartheta_{\max }$. In view of these generalization, we discuss the aperture dispersion and the shear dispersion and their relation to the shear correlation functions. Our results will be of practical use particularly for future cosmic shear surveys where highly precise measurements of the shear will become available and where control of systematics will be mandatory.
\end{abstract}

Key words. methods: statistical - gravitational lensing - cosmology: large-scale structure of the Universe - cosmology: dark matter

\section{Introduction}

The weak gravitational lensing effect by the large-scale matter distribution in the Universe is recognized as one of the most powerful cosmological probes (see Mellier 1999; Bartelmann \& Schneider 2001; Refregier 2003; Schneider 2006 for reviews). First observed in 2000 (Bacon et al. 2000; Kaiser et al. 2000; van Waerbeke et al. 2000; Wittman et al. 2000), this cosmic shear effect has advanced tremendously over the past few years (see, e.g., van Waerbeke et al. 2001, 2002, 2005; Maoli et al. 2001; Jarvis et al. 2003, 2005; Hoekstra et al. 2002, 2005; Bacon et al. 2003; Semboloni et al. 2005), and large ongoing and planned surveys will turn cosmic shear into an important tool for cosmology.

In order for satisfying its promises, systematic errors in cosmic shear measurements must be carefully controlled. Quite a few of those possible systematics have been identified, including the uncertainties in the overall shear calibration resulting from the correction of image smearing by seeing (e.g., Huterer et al. 2006) and uncertainties in the redshift distribution of source galaxies (e.g., Ma et al. 2006).

One of the important checks for possible systematic effects consists in investigating the measured shear for B-modes (Crittenden et al. 2002; hereafter C02), and most of the more recent cosmic shear studies perform such an analysis.

^ Founded by merging of the Sternwarte, Radioastronomisches Institut and Institut für Astrophysik und Extraterrestrische Forschung der Universität Bonn.
Gravitational lensing effects cause a shear field which, to leading order, only contains E-modes. The fact that early cosmic shear measurements contained a significant B-mode contribution of the shear was interpreted as remaining systematics. As has been pointed out by several groups (e.g., Heavens et al. 2000; Crittenden et al. 2001; Croft \& Metzler 2000; Catelan et al. 2000; Jing 2002), B-modes in the observed shear field can be caused by intrinsic alignments of source galaxies. This effect can be controlled in principle if sufficiently accurate photometric redshift information about the source galaxies is available (King \& Schneider 2002, 2003; Heymans \& Heavens 2003), but this is not the case for presently available surveys. In addition, B-modes are caused by gravitational lensing due to the redshift clustering of source galaxies, but the resulting signal is very small (Schneider et al. 2002, hereafter S02). If there are inhomogeneous systematic effects, such as a spatially dependent shear calibration error, B-modes would also be generated (Guzik \& Bernstein 2005).

The separation of the shear field into E- and B-modes is usually performed at the level of second-order statistics. Two standard methods for this have been established in the literature. One of them is the aperture dispersion statistics (Schneider et al. 1998) which is most conveniently expressed as an integral over the shear two-point correlation functions $\xi_{ \pm}$(see $\mathrm{C} 02$; S02). The second method is the calculation of shear correlation functions which contain only E- or B-modes (C02; S02). As was recently shown by Kilbinger et al. (2006), these two methods suffer from the fundamental problem that in order to apply them, one would 
in principle need to measure the shear correlation functions to zero separation (in the case of the aperture dispersion) or to infinite separation (for the E/B-mode correlation). Both of these measurements are impossible, as pairs of galaxy images blend if they have too low an angular separation, and the finite size of observational fields prevents measurements at arbitrary separations. This problem of principle becomes a problem in practice if one studies the presence of B-modes on small and large angular scales. In fact, as Kilbinger et al. (2006) have shown, the lack of knowledge of the shear correlation functions on scales below $\vartheta_{\text {min }}$ affects the aperture dispersion on scales below $\sim 15 \vartheta_{\text {min }}$ at more than the $\sim 10 \%$ level, and to have a less than $1 \%$ systematic bias, one needs radii for the aperture dispersion larger than $\sim 40 \vartheta_{\text {min }}$. Obviously, both for the diagnostics for the presence of B-modes, and for the use of the aperture dispersion for precision shear measurements, this systematic bias presents a problem.

In this paper, we present methods for overcoming this problem of principle, by defining integrals over the shear correlation functions which (i) extend over the finite range $\vartheta_{\min } \leq \vartheta \leq \vartheta_{\max }$ where the range of integration can be chosen arbitrarily, and which (ii) provide a clean separation of the shear signal into Eand B-modes. The first set of such integrals is termed the circle statistics; it is defined as the correlation of the mean tangential and cross-component of the shear on two circles with different radius. As we will show in Sect. 2, this correlation can be written in terms of integrals over the shear correlation function. Since the mean tangential (cross) shear on a circle depends solely on the E- (B-) mode of the shear field, this provides a clean separation of both modes. In Sect. 3 we consider the correlation of the mean tangential and cross shear over two rings which can as well be written in terms of integrals over the shear correlation functions $\xi_{ \pm}$. The relation of these new statistics to the aperture mass is briefly discussed in Sect. 4 . We then present a generalization of the circle and ring statistics in Sect. 5 which allows the choice of very general weighted integrals over the shear correlation functions that provide clear separation into E- and B-modes. We briefly conclude in Sect. 6.

\section{The circle statistics}

\subsection{Definition}

Consider a circle of radius $\theta$ around the origin and define the mean of the tangential and cross component of the shear on this circle, $C_{\mathrm{t}}$ and $C_{\times}$, respectively, measured with respect to the center,

$$
\begin{aligned}
C(\theta) & =C_{\mathrm{t}}(\theta)+\mathrm{i} C_{\times}(\theta) \\
& =\frac{1}{2 \pi} \int_{0}^{2 \pi} \mathrm{d} \varphi\left(\gamma_{\mathrm{t}}+\mathrm{i} \gamma_{\times}\right)(\theta, \varphi) .
\end{aligned}
$$

The tangential and cross-components of the shear are related to the Cartesian shear components $\gamma^{\mathrm{c}}=\gamma_{1}+\mathrm{i} \gamma_{2}$ through a rotation,

$\gamma_{\mathrm{t}}+\mathrm{i} \gamma_{\times}=-\gamma^{\mathrm{c}} \mathrm{e}^{-2 \mathrm{i} \varphi}$,

where $\varphi$ describes the polar angle on the circle. As is well known (see, e.g., C02; S02), $C_{\mathrm{t}}$ is sensitive only to the E-mode of the shear field, whereas $C_{\times}$is sensitive only to the B-mode.

We next consider the correlation between the mean shear on two concentric circles with radii $\theta_{1}$ and $\theta_{2}$,

$$
\begin{aligned}
\left\langle C\left(\theta_{1}\right) C\left(\theta_{2}\right)\right\rangle= & \int_{0}^{2 \pi} \frac{\mathrm{d} \varphi_{1}}{2 \pi} \int_{0}^{2 \pi} \frac{\mathrm{d} \varphi_{2}}{2 \pi} \mathrm{e}^{-2 \mathrm{i}\left(\varphi_{1}+\varphi_{2}\right)} \\
& \times\left\langle\gamma^{\mathrm{c}}\left(\theta_{1}, \varphi_{1}\right) \gamma^{\mathrm{c}}\left(\theta_{2}, \varphi_{2}\right)\right\rangle,
\end{aligned}
$$

where we made use of (2). Correspondingly, we define the correlator

$$
\begin{aligned}
\left\langle C\left(\theta_{1}\right) C^{*}\left(\theta_{2}\right)\right\rangle= & \int_{0}^{2 \pi} \frac{\mathrm{d} \varphi_{1}}{2 \pi} \int_{0}^{2 \pi} \frac{\mathrm{d} \varphi_{2}}{2 \pi} \mathrm{e}^{-2 \mathrm{i}\left(\varphi_{1}-\varphi_{2}\right)} \\
& \times\left\langle\gamma^{\mathrm{c}}\left(\theta_{1}, \varphi_{1}\right) \gamma^{\mathrm{c} *}\left(\theta_{2}, \varphi_{2}\right)\right\rangle,
\end{aligned}
$$

where the asterisk denotes complex conjugation. Expanding the correlators then yields

$$
\left\langle C\left(\theta_{1}\right) C\left(\theta_{2}\right)\right\rangle=\left\langle C_{\mathrm{t}}\left(\theta_{1}\right) C_{\mathrm{t}}\left(\theta_{2}\right)\right\rangle-\left\langle\mathcal{C}_{\times}\left(\theta_{1}\right) C_{\times}\left(\theta_{2}\right)\right\rangle,
$$

and the imaginary part vanishes if we assume that the shear field is parity invariant (Schneider 2003); this assumption is equivalent to the requirement that the mixed shear correlator $\left\langle\gamma_{\mathrm{t}} \gamma_{\times}\right\rangle \equiv 0$. Similarly,

$\left\langle C\left(\theta_{1}\right) C^{*}\left(\theta_{2}\right)\right\rangle=\left\langle C_{\mathrm{t}}\left(\theta_{1}\right) C_{\mathrm{t}}\left(\theta_{2}\right)\right\rangle+\left\langle C_{\times}\left(\theta_{1}\right) C_{\times}\left(\theta_{2}\right)\right\rangle$,

so that the separation into E- and B-modes is achieved with

$$
\begin{aligned}
& \left\langle C_{\mathrm{t}}\left(\theta_{1}\right) \mathcal{C}_{\mathrm{t}}\left(\theta_{2}\right)\right\rangle=\frac{1}{2}\left[\left\langle C\left(\theta_{1}\right) C^{*}\left(\theta_{2}\right)\right\rangle+\left\langle C\left(\theta_{1}\right) C\left(\theta_{2}\right)\right\rangle\right], \\
& \left\langle C_{\times}\left(\theta_{1}\right) C_{\times}\left(\theta_{2}\right)\right\rangle=\frac{1}{2}\left[\left\langle C\left(\theta_{1}\right) C^{*}\left(\theta_{2}\right)\right\rangle-\left\langle C\left(\theta_{1}\right) C\left(\theta_{2}\right)\right\rangle\right] .
\end{aligned}
$$

\subsection{Relation to the shear correlation functions}

The shear correlators that occur in (3) and (4) can be expressed in terms of the usual shear correlation function $\xi_{ \pm}$by rotating the Cartesian shear into tangential and cross components relative to the separation direction of the two points with polar coordinates $\left(\theta_{1}, \varphi_{1}\right)$ and $\left(\theta_{2}, \varphi_{2}\right)$. We then find that

$\left\langle\gamma^{\mathrm{c}}\left(\theta_{1}, \varphi_{1}\right) \gamma^{\mathrm{c}}\left(\theta_{2}, \varphi_{2}\right)\right\rangle=\mathrm{e}^{4 \mathrm{i} \varphi} \xi_{-}(\vartheta)$,

$\left\langle\gamma^{\mathrm{c}}\left(\theta_{1}, \varphi_{1}\right) \gamma^{\mathrm{c} *}\left(\theta_{2}, \varphi_{2}\right)\right\rangle=\xi_{+}(\vartheta)$

where $\vartheta$ and $\varphi$ are the polar coordinates of the separation vector,

$\vartheta \mathrm{e}^{\mathrm{i} \varphi}=\theta_{2} \mathrm{e}^{\mathrm{i} \varphi_{2}}-\theta_{1} \mathrm{e}^{\mathrm{i} \varphi_{1}}$.

More explicitly, the separation is

$\vartheta^{2}=\theta_{1}^{2}+\theta_{2}^{2}-2 \theta_{1} \theta_{2} \cos (\Delta \varphi)$

where $\Delta \varphi=\varphi_{2}-\varphi_{1}$ is the difference of the polar angle of the two points under consideration. In the calculation of (3) we need the square of the expression

$\mathrm{e}^{2 \mathrm{i} \varphi} \mathrm{e}^{-\mathrm{i}\left(\varphi_{1}+\varphi_{2}\right)}=\frac{\theta_{1}^{2} \mathrm{e}^{-\mathrm{i} \Delta \varphi}+\theta_{2}^{2} \mathrm{e}^{\mathrm{i} \Delta \varphi}-2 \theta_{1} \theta_{2}}{\vartheta^{2}}$.

The integrand in (3) then depends only on the difference $\Delta \varphi$ of the polar angles, so that one of the angular integrals can be carried out. Furthermore, we note that the integral is real, since the imaginary part of the integrand is an odd function of $\Delta \varphi$. This then allows us to write the correlator of the circle statistics as

$$
\begin{aligned}
\left\langle C\left(\theta_{1}\right) C\left(\theta_{2}\right)\right\rangle= & \int_{0}^{\pi} \frac{\mathrm{d} \Delta \varphi}{\pi} \frac{\xi_{-}(\vartheta)}{\vartheta^{4}}\left[\left(\theta_{1}^{4}+\theta_{2}^{4}\right) \cos (2 \Delta \varphi)\right. \\
& \left.-4 \theta_{1} \theta_{2}\left(\theta_{1}^{2}+\theta_{2}^{2}\right) \cos (\Delta \varphi)+6 \theta_{1}^{2} \theta_{2}^{2}\right] .
\end{aligned}
$$

We can now transform the integral into one over the separation $\vartheta$. Using (12) we find for the transformation of variables

$\mathrm{d} \Delta \varphi=\frac{2 \vartheta \mathrm{d} \vartheta}{\sqrt{\left(\theta_{1}+\theta_{2}\right)^{2}-\vartheta^{2}} \sqrt{\vartheta^{2}-\left(\theta_{2}-\theta_{1}\right)^{2}}}$ 
The $\vartheta$-integration then extends from $\theta_{2}-\theta_{1}$ to $\theta_{1}+\theta_{2}$, where we set $\theta_{1} \leq \theta_{2}$ without loss of generality. Then, the final expression can be written as

$\left\langle C\left(\theta_{1}\right) C\left(\theta_{2}\right)\right\rangle=\int_{\theta_{2}-\theta_{1}}^{\theta_{1}+\theta_{2}} \frac{\mathrm{d} \vartheta}{\vartheta} \xi_{-}(\vartheta) Y_{-}\left(\frac{\vartheta}{\theta_{2}}, \frac{\theta_{1}}{\theta_{2}}\right)$,

where the dimensionless function $Y_{-}$is

$$
\begin{aligned}
Y_{-}(x, \eta)= & \frac{1}{\pi \eta^{2} x^{2} \sqrt{(1+\eta)^{2}-x^{2}} \sqrt{x^{2}-(1-\eta)^{2}}} \\
& \times\left[\left(1-x^{2}\right)^{2}-2 \eta^{2}\left(2-x^{2}\right)+\eta^{4}\left(6+2 x^{2}+x^{4}\right)\right. \\
& \left.-2 \eta^{6}\left(2+x^{2}\right)+\eta^{8}\right] .
\end{aligned}
$$

In a similar way, we obtain for the other correlator (4)

$$
\begin{aligned}
\left\langle C\left(\theta_{1}\right) C^{*}\left(\theta_{2}\right)\right\rangle & =\int_{0}^{\pi} \frac{\mathrm{d} \Delta \varphi}{\pi} \xi_{+}(\vartheta) \cos (2 \Delta \varphi) \\
& =\int_{\theta_{2}-\theta_{1}}^{\theta_{1}+\theta_{2}} \frac{\mathrm{d} \vartheta}{\vartheta} \xi_{+}(\vartheta) Y_{+}\left(\frac{\vartheta}{\theta_{2}}, \frac{\theta_{1}}{\theta_{2}}\right),
\end{aligned}
$$

with

$$
Y_{+}(x, \eta)=\frac{x^{2}\left[\left(1-x^{2}\right)^{2}-2 \eta^{2} x^{2}+\eta^{4}\right]}{\eta^{2} \pi \sqrt{(1+\eta)^{2}-x^{2}} \sqrt{x^{2}-(1-\eta)^{2}}} .
$$

We therefore get for the E-mode of the circle statistics

$$
\begin{aligned}
\left\langle C_{\mathrm{t}}\left(\theta_{1}\right) \mathcal{C}_{\mathrm{t}}\left(\theta_{2}\right)\right\rangle= & \int_{\theta_{2}-\theta_{1}}^{\theta_{1}+\theta_{2}} \frac{\mathrm{d} \vartheta}{2 \vartheta}\left[\xi_{+}(\vartheta) Y_{+}\left(\frac{\vartheta}{\theta_{2}}, \frac{\theta_{1}}{\theta_{2}}\right)\right. \\
& \left.+\xi_{-}(\vartheta) Y_{-}\left(\frac{\vartheta}{\theta_{2}}, \frac{\theta_{1}}{\theta_{2}}\right)\right],
\end{aligned}
$$

and the corresponding B-mode reads

$$
\begin{aligned}
\left\langle C_{\times}\left(\theta_{1}\right) C_{\times}\left(\theta_{2}\right)\right\rangle= & \int_{\theta_{2}-\theta_{1}}^{\theta_{1}+\theta_{2}} \frac{\mathrm{d} \vartheta}{2 \vartheta}\left[\xi_{+}(\vartheta) Y_{+}\left(\frac{\vartheta}{\theta_{2}}, \frac{\theta_{1}}{\theta_{2}}\right)\right. \\
& \left.-\xi_{-}(\vartheta) Y_{-}\left(\frac{\vartheta}{\theta_{2}}, \frac{\theta_{1}}{\theta_{2}}\right)\right] .
\end{aligned}
$$

Hence, we have obtained a statistics which is able to perform the E-/B-mode separation of the shear correlation functions on a finite interval in angular separation, $\theta_{2}-\theta_{1} \leq \vartheta \leq \theta_{1}+\theta_{2}$. In particular, it avoids the use of correlation functions towards zero separation which, as we argued before, are difficult or impossible to be obtained from observations and whose neglect causes artificial E-/B-mode mixing in the aperture statistics.

However, the circle statistic is not very practical, since the functions $Y_{ \pm}$have a $\vartheta^{-1 / 2}$ singularity at both integration boundaries. This singularity has a purely geometrical origin, in that at the minimum and maximum separation of points on the two circles, the circles are "mutually parallel", so that pairs with these two extremum separations are particularly frequent. Applying the foregoing equations to observed correlation functions with their noise will therefore yield estimates for the circle statistics which will be dominated by the noise just at the boundaries. It it is therefore desirable to find an expression for the E-/B-mode separation on a finite interval which does not contain such a singularity. This will be provided by the ring statistics, which is discussed in Sect. 3.

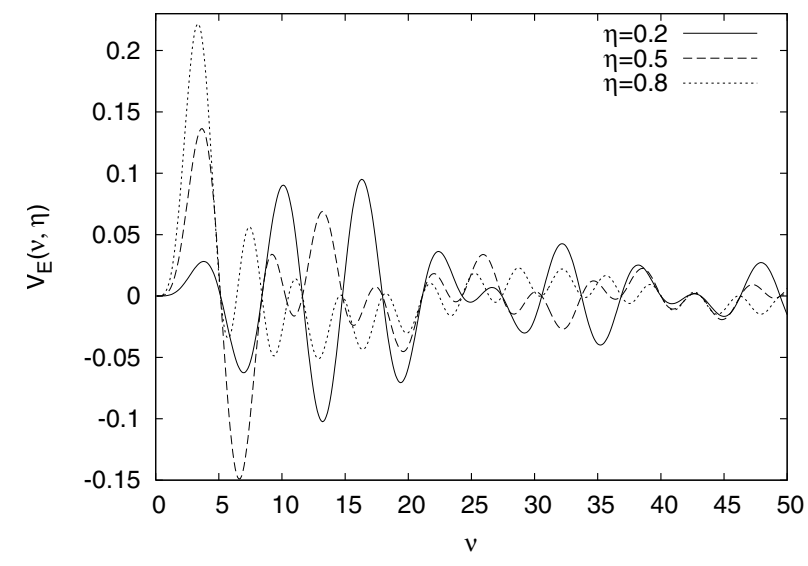

Fig. 1. The filter function $\mathcal{V}_{\mathrm{E}}$ relating the power spectrum to the circle statistics, see Eq. (23), for three different choices of the ratio of radii $\eta=\theta_{1} / \theta_{2}$.

\subsection{Relation to the power spectrum}

If we assume that the shear field is caused by gravitational lensing, no B-modes are present. The shear field then is a pure E-mode field which derives from the underlying surface mass density $\kappa$ (we use standard lensing notation here). The power spectrum $P(\ell)$ of the $\kappa$ field then specifies the second-order statistics of the shear field, in particular the correlation functions. We have (see, e.g., Kaiser 1992)

$\xi_{ \pm}(\vartheta)=\int_{0}^{\infty} \frac{\mathrm{d} \ell \ell}{2 \pi} P(\ell) J_{0,4}(\vartheta \ell)$

where the $J_{n}$ are Bessel functions of the first kind. Inserting this expression into the E-mode circle statistics (20) yields

$\left\langle C_{\mathrm{t}}\left(\theta_{1}\right) C_{\mathrm{t}}\left(\theta_{2}\right)\right\rangle=\int_{0}^{\infty} \frac{\mathrm{d} \ell \ell}{2 \pi} P(\ell) \mathcal{V}_{\mathrm{E}}\left(\ell \theta_{2}, \frac{\theta_{1}}{\theta_{2}}\right)$,

where

$\nu_{\mathrm{E}}(v, \eta)=\int_{1-\eta}^{1+\eta} \frac{\mathrm{d} x}{2 x}\left[J_{0}(v x) Y_{+}(x, \eta)+J_{4}(v x) Y_{-}(x, \eta)\right]$.

Hence, the function $\mathcal{V}_{\mathrm{E}}(v, \eta)$ describes the filter function with which the circle statistics is related to the lensing power spectrum; it depends on the ratio $\eta$ of the two radii. The filter function is plotted in Fig. 1 for three values of $\eta$. It shows an oscillating behavior which reflects the oscillations of the Bessel functions.

The behavior of $\mathcal{V}_{\mathrm{E}}$ for small and large values of $v$ can be obtained analytically. Considering small $v$ first, we can expand the Bessel functions in the foregoing equation. The leading-order term in $J_{4}$ is $(v x)^{4}$, whereas the expansion of $J_{0}$ contains terms proportional to $(v x)^{0}$ and $(v x)^{2}$. However, we find that

$\int_{1-\eta}^{1+\eta} \frac{\mathrm{d} x}{2 x} Y_{+}(x, \eta)=0=\int_{1-\eta}^{1+\eta} \frac{\mathrm{d} x}{2 x} x^{2} Y_{+}(x, \eta)$

so that the leading-order term from the $J_{0}$ part is also $\propto v^{4}$. Together, we conclude that $\mathcal{V}_{\mathrm{E}}(v, \eta) \propto v^{4}$ for small $v$, as can also be seen in Fig. 1.

In the opposite case of large $v$, the behavior of the integral for $\mathcal{V}_{\mathrm{E}}(v, \eta)$ is dominated by the $1 / \sqrt{x}$-singularity of the functions $Y_{ \pm}$at both integration limits. Hence, the asymptotic behavior of $\mathcal{V}_{\mathrm{E}}(v, \eta)$ can be obtained from an integral with very similar properties,

$\int_{-x_{0}}^{x_{0}} \mathrm{~d} x \frac{J_{0}(v x)}{\sqrt{x_{0}^{2}-x^{2}}}=\pi J_{0}^{2}\left(\frac{v x_{0}}{2}\right)$ 
and

$\int_{-x_{0}}^{x_{0}} \mathrm{~d} x \frac{J_{4}(v x)}{\sqrt{x_{0}^{2}-x^{2}}}=\pi J_{2}^{2}\left(\frac{v x_{0}}{2}\right)$.

Thus, for large $v$ we expect $\mathcal{V}_{\mathrm{E}}(v, \eta)$ to be an oscillating function, where the amplitude of the oscillation decreases as $v^{-1}$. Again, this behavior is seen in Fig. 1 .

The vanishing of the B-mode implies that

$$
\begin{aligned}
\mathcal{V}_{\mathrm{B}}(v, \eta) & =\int_{1-\eta}^{1+\eta} \frac{\mathrm{d} x}{2 x}\left[J_{0}(v x) Y_{+}(x, \eta)-J_{4}(v x) Y_{-}(x, \eta)\right] \\
& \equiv 0
\end{aligned}
$$

for all values of $v$ and arbitrary $0<\eta<1$. As a consequence, in the absence of B-modes, the circle statistics can be calculated from $\xi_{+}$and $\xi_{-}$separately, since both terms occurring in (20) are then equal.

\section{The ring statistics}

Instead of the shear on a circle, we consider here the shear inside a ring and define

$\mathcal{R}=\mathcal{R}_{\mathrm{t}}+\mathrm{i} \mathcal{R}_{\times}=\int_{\zeta_{1}}^{\zeta_{2}} \mathrm{~d} \theta W(\theta) C(\theta)$,

where the integral extends over the annulus $\zeta_{1} \leq \theta \leq \zeta_{2}$, and $W(\theta)$ is a weight function which we choose to be normalized to unity,

$\int_{\zeta_{1}}^{\zeta_{2}} \mathrm{~d} \theta W(\theta)=1$.

As was true for the circle statistics, the real and imaginary parts of $\mathcal{R}$ are solely due to E-modes and B-modes, respectively. We now consider the correlator of two such concentric rings, one with $\zeta_{1} \leq \theta \leq \zeta_{2}$ as before, the other with $\zeta_{3} \leq \theta \leq \zeta_{4}$. If $\zeta_{i}<\zeta_{j}$ for all $i<j$, as we shall assume from here on, the rings are non-overlapping. The maximum and minimum separation of points from one ring to the other are $\vartheta_{\max }=\zeta_{2}+\zeta_{4} \equiv \Psi$ and $\vartheta_{\text {min }}=\zeta_{3}-\zeta_{2} \equiv \eta \Psi$, respectively. The correlator reads

$$
\begin{aligned}
\langle\mathcal{R R}\rangle= & \int_{\zeta_{1}}^{\zeta_{2}} \mathrm{~d} \theta_{1} W_{1}\left(\theta_{1}\right) \int_{\zeta_{3}}^{\zeta_{4}} \mathrm{~d} \theta_{2} W_{2}\left(\theta_{2}\right)\left\langle C\left(\theta_{1}\right) C\left(\theta_{2}\right)\right\rangle \\
= & \int_{\zeta_{1}}^{\zeta_{2}} \mathrm{~d} \theta_{1} W_{1}\left(\theta_{1}\right) \int_{\zeta_{3}}^{\zeta_{4}} \mathrm{~d} \theta_{2} W_{2}\left(\theta_{2}\right) \\
& \times \int_{\theta_{2}-\theta_{1}}^{\theta_{1}+\theta_{2}} \frac{\mathrm{d} \vartheta}{\vartheta} \xi_{-}(\vartheta) Y_{-}\left(\frac{\vartheta}{\theta_{2}}, \frac{\theta_{1}}{\theta_{2}}\right) .
\end{aligned}
$$

Interchanging the order of integration, this correlator can be written in the form

$\langle\mathcal{R R}\rangle=\int_{\eta}^{1} \frac{\mathrm{d} x}{x} \xi_{-}(x \Psi) Z_{-}(x, \eta)$,

where $\eta=\vartheta_{\min } / \vartheta_{\max }$ is the ratio of minimum and maximum separation of points between the two rings, and the function $Z_{-}(x, \eta)$ can be calculated explicitly by changing the integration order in (26); this is done explicitly in (A.1). In general, the resulting form of the function $Z_{-}$will be complicated, due to the numerous case distinctions in the range of integrations. However, if the ratios of the ring radii are chosen to take on specific values, the number of case distinctions is minimized, and the calculation of

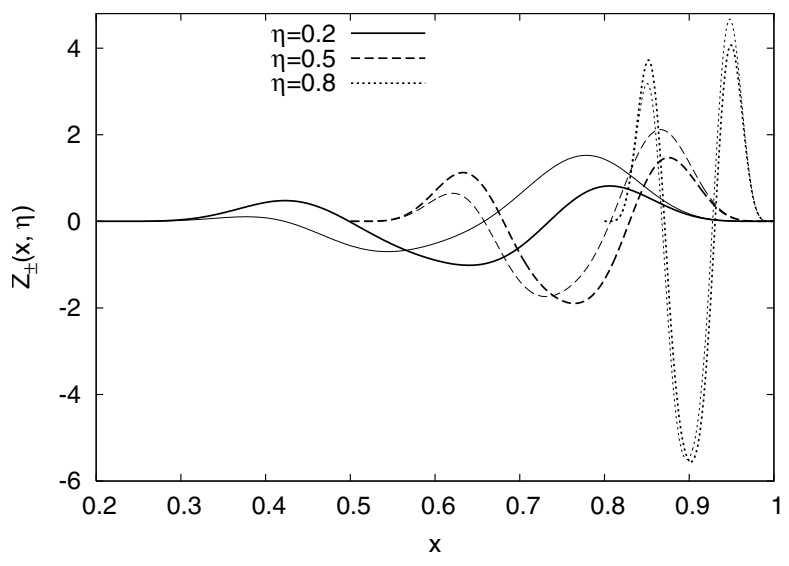

Fig. 2. The functions $Z_{+}$(thick curves) and $Z_{-}$(thin curves) as functions of the angular separation scaled by the maximum separation, $x=\vartheta / \vartheta_{\max }$, for three different values of the ratio $\eta=\vartheta_{\min } / \vartheta_{\max }$. The specific choice for the ring boundaries (28) and weight functions (30) was assumed.

the function $Z_{-}(x, \eta)$ simplifies considerably. In the appendix, we provide explicit formulae for $Z_{-}$for the following choice:

$\zeta_{1}=\frac{1-\eta}{8} \Psi ; \zeta_{2}=\frac{3(1-\eta)}{8} \Psi$,
$\zeta_{3}=\frac{5 \eta+3}{8} \Psi ; \zeta_{4}=\frac{3 \eta+5}{8} \Psi$.

This choice still allows the freedom to choose the range $\eta \Psi \leq$ $\vartheta \leq \Psi$ over which the correlation function is probed in the ring statistics. In analogy, the other correlator involving $\xi_{+}$reads

$\left\langle\mathcal{R R}^{*}\right\rangle=\int_{\eta}^{1} \frac{\mathrm{d} x}{x} \xi_{+}(x \Psi) Z_{+}(x, \eta)$.

The weight funtions $W_{i}$ can be chosen arbitrarily; the smoother they are near their respective edges, the smoother the functions $Z_{ \pm}$. To be specific, we will choose the weight functions to be polynomials in their range of definition, and which have continuous first derivatives at their boundary,

$W_{1}\left(\theta_{1}\right)=\frac{30\left(\theta_{1}-\zeta_{1}\right)^{2}\left(\zeta_{2}-\theta_{1}\right)^{2}}{\left(\zeta_{2}-\zeta_{1}\right)^{5}}$,
$W_{2}\left(\theta_{2}\right)=\frac{30\left(\theta_{2}-\zeta_{3}\right)^{2}\left(\zeta_{4}-\theta_{2}\right)^{2}}{\left(\zeta_{4}-\zeta_{3}\right)^{5}}$.

In Fig. 2 we have plotted the functions $Z_{ \pm}$for three values of $\eta$. Both functions $Z_{+}$and $Z_{-}$have two roots in the interval $\eta<x<1$, starting off with positive values near the edges of their range of support. We will explain this qualitative behavior in Sect. 5.2 below.

The E- and B-modes of the ring statistics are given by the combinations

$$
\begin{aligned}
\langle\mathcal{R} \mathcal{R}\rangle_{\mathrm{E}} & =\frac{1}{2}\left(\left\langle\mathcal{R} \mathcal{R}^{*}\right\rangle+\langle\mathcal{R} \mathcal{R}\rangle\right), \\
& =\int_{\eta}^{1} \frac{\mathrm{d} x}{2 x}\left[\xi_{+}(x \Psi) Z_{+}(x, \eta)+\xi_{-}(x \Psi) Z_{-}(x, \eta)\right], \\
\langle\mathcal{R} \mathcal{R}\rangle_{\mathrm{B}} & =\frac{1}{2}\left(\left\langle\mathcal{R} \mathcal{R}^{*}\right\rangle-\langle\mathcal{R} \mathcal{R}\rangle\right) \\
& =\int_{\eta}^{1} \frac{\mathrm{d} x}{2 x}\left[\xi_{+}(x \Psi) Z_{+}(x, \eta)-\xi_{-}(x \Psi) Z_{-}(x, \eta)\right] .
\end{aligned}
$$




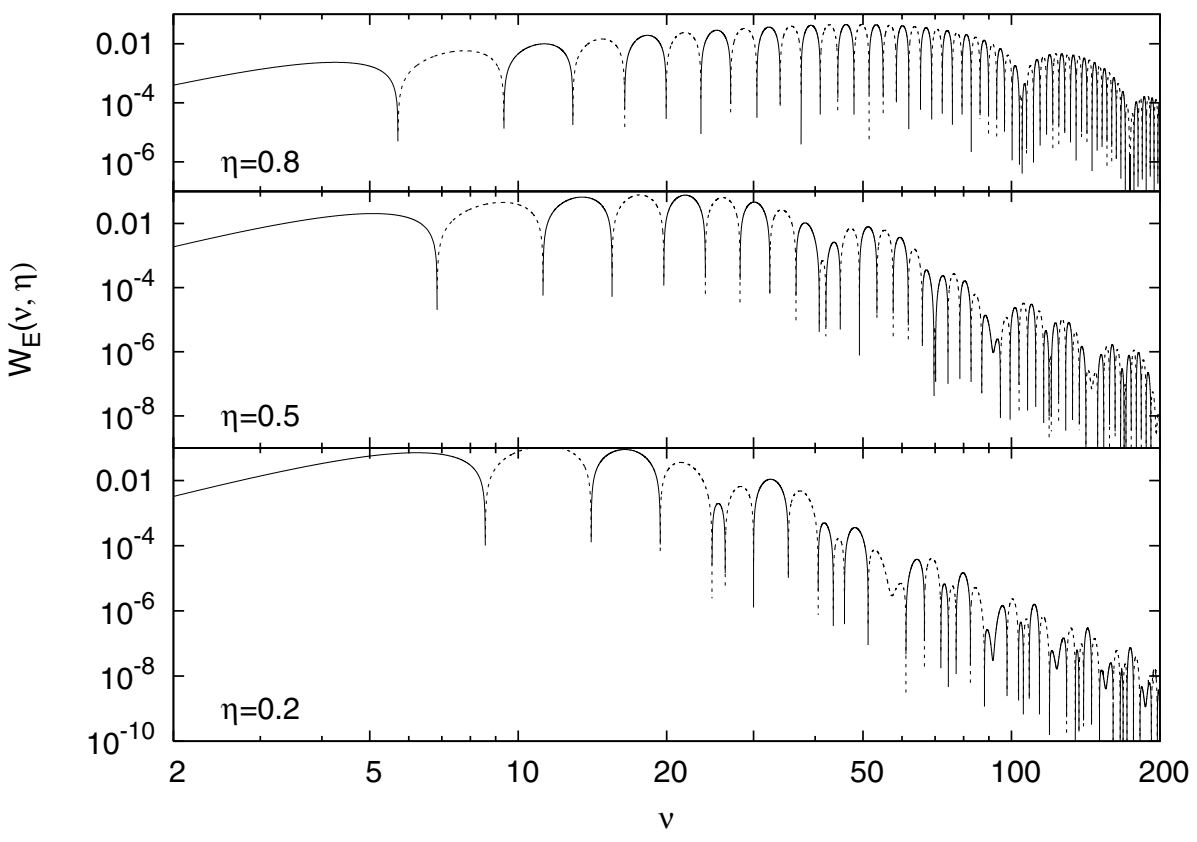

Fig. 3. The filter function $\mathcal{W}_{\mathrm{E}}$ relating the power spectrum to the ring statistics, see Eq. (32), for three different choices of the ratio of radii $\eta=\theta_{1} / \theta_{2}$. Dotted curves indicate negative values.
In case the shear field derives from gravitational lensing, its $\mathrm{B}$-modes vanish, and the ring statistics $\langle\mathcal{R} \mathcal{R}\rangle_{\mathrm{E}}$ can be expressed in terms of the power spectrum $P(\ell)$ of the projected surface mass density. In analogy to the circle statistics, we obtain

$\langle\mathcal{R R}\rangle_{\mathrm{E}}=\int_{0}^{\infty} \frac{\mathrm{d} \ell \ell}{2 \pi} P(\ell) \mathcal{W}_{\mathrm{E}}(\ell \Psi, \eta)$,

where

$\mathcal{W}_{\mathrm{E}}(v, \eta)=\int_{\eta}^{1} \frac{\mathrm{d} x}{2 x}\left[J_{0}(v x) Z_{+}(x, \eta)+J_{4}(v x) Z_{-}(x, \eta)\right]$.

In Fig. 3 we have plotted the function $\mathcal{W}_{\mathrm{E}}(v, \eta)$ which is the filter function that relates the power spectrum to the ring statistics, for three different values of $\eta$. As we can see, $\mathcal{W}_{\mathrm{E}}$ is a smooth function for small $v$, whereas it strongly oscillates for larger values of $v$. Asymptotically, $\mathcal{W}_{\mathrm{E}}(v, \eta)$ goes as $v^{4}$ for small $v$, and the amplitude of oscillations falls off as $v^{-7}$ for large $v$.

The ring statistics $\langle\mathcal{R} \mathcal{R}\rangle_{\mathrm{E}}$ is plotted in Fig. 4, for a $\Lambda \mathrm{CDM}$ model with $\sigma_{8}=0.85$ and a mean redshift of source galaxies of $\bar{z} \approx 1.5$. For this calculation, the non-linear power spectrum according to the prescription of Smith et al. (2003) has been used. We see that $\langle\mathcal{R} \mathcal{R}\rangle_{\mathrm{E}}$ has a very small amplitude which decreases for larger $\eta$. Hence, the narrower the interval is over which the correlation functions are considered, the smaller is the measured shear signal. For all values of $\eta$, the signal of $\langle\mathcal{R R}\rangle_{\mathrm{E}}$ is much smaller than the characteristic values of the shear correlation functions. The mathematical reason for this can be seen in Fig. 2 in combination with (31): to derive $\langle\mathcal{R R}\rangle_{\mathrm{E}}$, the shear correlation is integrated over the functions $Z_{ \pm}$which has two roots in their range of support. Another way to see this is by considering the function $\mathcal{W}_{\mathrm{E}}$ (Fig. 3) in connection with (32). Due to its oscillating behavior, the resulting integral in (32) is expected to be small. The narrower the ring is (i.e., the larger $\eta$ ), the more oscillations does the function $\mathcal{W}_{\mathrm{E}}$ show near its broad maximum, and the larger is the near-cancellation of power in the integral (32).

The vanishing of the B-mode then implies that

$$
\begin{aligned}
\mathcal{W}_{\mathrm{B}}(v, \eta) & =\int_{\eta}^{1} \frac{\mathrm{d} x}{2 x}\left[J_{0}(v x) Z_{+}(x, \eta)-J_{4}(v x) Z_{-}(x, \eta)\right] \\
& \equiv 0
\end{aligned}
$$

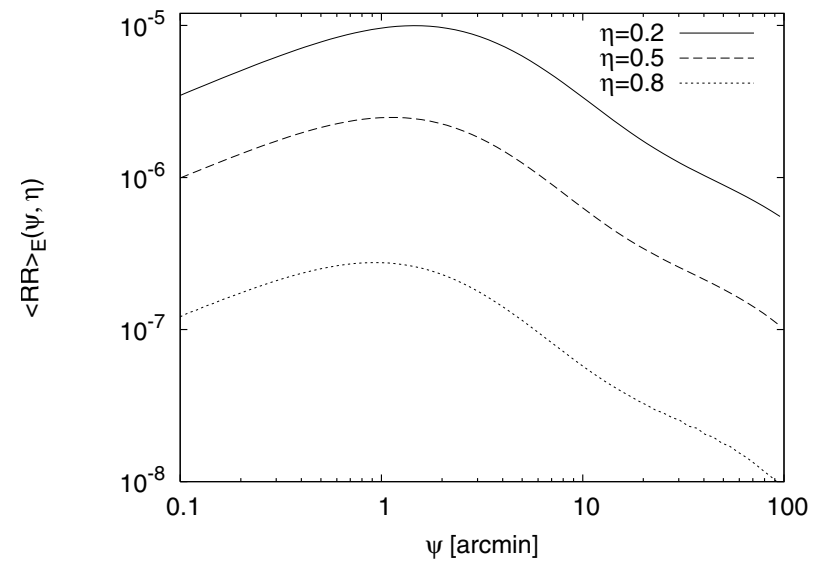

Fig. 4. The ring statistics $\langle\mathcal{R} \mathcal{R}\rangle_{\mathrm{E}}$ (32) as a function of the maximum separation $\Psi=\vartheta_{\max }$ for which the correlation functions need to be known, for three different ratios $\eta=\vartheta_{\min } / \vartheta_{\max }$.

for all values of $v$. We have checked numerically that the functions $Z_{ \pm}$indeed satisfy this relation.

\section{Relation to the aperture statistics}

The definition (24) of the ring statistics is very similar to that of the aperture mass (Kaiser 1995; Schneider 1996), which is defined as

$M_{\text {ap }}=\int \mathrm{d}^{2} \vartheta \kappa(\boldsymbol{\vartheta}) U(|\boldsymbol{\vartheta}|)$,

where $U$ is a radial weight function and the integral extends over the support of $U$. Provided $U$ is a compensated filter function, $\int \mathrm{d} \vartheta \vartheta U(\vartheta)=0, M_{\text {ap }}$ can be written in terms of the tangential shear,

$M_{\text {ap }}=\int \mathrm{d}^{2} \vartheta \gamma_{\mathrm{t}}(\boldsymbol{\vartheta}) Q(|\boldsymbol{\vartheta}|)$,

where the weight function $Q$ is related to $U$ by

$Q(\theta)=\frac{2}{\theta^{2}} \int_{0}^{\theta} \mathrm{d} \vartheta \vartheta U(\vartheta)-U(\theta)$ 
The definition of $\mathcal{R}$ is equivalent to (35) in the absence of B-modes, if we set $W(\theta)=2 \pi \theta Q(\theta)$. It is therefore interesting to see whether the ring statistics can be expressed also in terms of the surface mass density. This indeed is the case, since (36) can be reversed. Multiplying (36) with $\theta^{2}$ and differentiating with respect to $\theta$ yields the differential equation

$U^{\prime}=-Q^{\prime}-\frac{2}{\theta} Q$

which can be readily integrated, using the boundary condition $U(\theta) \rightarrow 0$ as $\theta \rightarrow \infty$ :

$U(\theta)=\int_{\theta}^{\infty} \frac{2 \mathrm{~d} \vartheta}{\vartheta} Q(\vartheta)-Q(\theta)$.

It is straightforward to check that $U$ is a compensated filter function. Furthermore, if $Q(\theta)=0$ for $\theta>\theta_{\max }$, then also $U(\theta)=0$ for $\theta>\theta_{\max }$. Therefore, there is an equivalent $U$-filter with which the ring statistics can be expressed in terms of the surface mass density; $U$ is a positive constant for $0 \leq \theta \leq \zeta_{1}$, then decreases and eventually becomes negative somewhere in the interval $\zeta_{1}<\theta<\zeta_{2}$, and gets zero again for $\theta \geq \zeta_{2}$.

\section{Generalization}

The relations (31) that define integrals over the shear correlation functions which are sensitive separately to E- and B-modes are very similar to analogous equations for the aperture dispersions (see S02; C02). We could easily find other forms of the function $Z_{ \pm}$by taking different choices of the weight functions $W_{1,2}$ entering the definition of the ring statistics.

Therefore, the question arises whether one can find a wide class of functions, analogous to $Z_{ \pm}$in (31) which provides a separation of the shear correlation into E- and B-modes. This question will be answered here.

\subsection{The general E/B-mode decomposition}

Thus, we define second-order shear statistics in the following form:

$E=\int_{0}^{\infty} \mathrm{d} \vartheta \vartheta\left[\xi_{+}(\vartheta) T_{+}(\vartheta)+\xi_{-}(\vartheta) T_{-}(\vartheta)\right]$,

$B=\int_{0}^{\infty} \mathrm{d} \vartheta \vartheta\left[\xi_{+}(\vartheta) T_{+}(\vartheta)-\xi_{-}(\vartheta) T_{-}(\vartheta)\right]$,

and look for pairs of function $T_{ \pm}(\vartheta)$ for which $E$ contains only E-modes, and $B$ contains only B-modes. In terms of the $\mathrm{E} / \mathrm{B}$-mode power spectra, the correlation functions read (see S02)

$\xi_{+}(\vartheta)=\int_{0}^{\infty} \frac{\mathrm{d} \ell \ell}{2 \pi} J_{0}(\ell \vartheta)\left[P_{\mathrm{E}}(\ell)+P_{\mathrm{B}}(\ell)\right]$,

$\xi_{-}(\vartheta)=\int_{0}^{\infty} \frac{\mathrm{d} \ell \ell}{2 \pi} J_{4}(\ell \vartheta)\left[P_{\mathrm{E}}(\ell)-P_{\mathrm{B}}(\ell)\right]$.

Inserting (40) into (39) and interchanging the order of integration, we obtain

$$
\begin{aligned}
E=\int_{0}^{\infty} \frac{\mathrm{d} \ell \ell}{2 \pi} & \left(P_{\mathrm{E}}(\ell)\left[t_{+}(\ell)+t_{-}(\ell)\right]\right. \\
& \left.+P_{\mathrm{B}}(\ell)\left[t_{+}(\ell)-t_{-}(\ell)\right]\right), \\
B=\int_{0}^{\infty} \frac{\mathrm{d} \ell \ell}{2 \pi} & \left(P_{\mathrm{E}}(\ell)\left[t_{+}(\ell)-t_{-}(\ell)\right]\right. \\
& \left.+P_{\mathrm{B}}(\ell)\left[t_{+}(\ell)+t_{-}(\ell)\right]\right),
\end{aligned}
$$

where we have defined the Hankel transform of the functions $T_{ \pm}$,

$$
\begin{aligned}
& t_{+}(\ell)=\int_{0}^{\infty} \mathrm{d} \vartheta \vartheta T_{+}(\vartheta) J_{0}(\ell \vartheta) \\
& t_{-}(\ell)=\int_{0}^{\infty} \mathrm{d} \vartheta \vartheta T_{-}(\vartheta) J_{4}(\ell \vartheta)
\end{aligned}
$$

Thus, the functions $E$ and $B$ contain only E- and B-modes, respectively, if and only if the two Hankel transforms are identical,

$t_{+}(\ell) \equiv t_{-}(\ell)$

Any pair of functions $T_{ \pm}$which satisfies (43) leads to a separation into E- and B-modes. The functions $Y_{ \pm}$for the circle statistics and the functions $Z_{ \pm}$for the ring statistics are special examples of this, as are the pairs of functions $T_{ \pm}$for the aperture statistics and $S_{ \pm}$for the shear dispersion, as defined in S02.

We will show next, by explicit construction, that one can choose one of the two functions, $T_{+}$or $T_{-}$, arbitrarily and the other function can be uniquely calculated such that (43) is satisfied. First we assume that the function $T_{-}$is chosen; then we calculate $T_{+}$from the inverse Hankel transform,

$T_{+}(\vartheta)=\int_{0}^{\infty} \mathrm{d} \ell \ell J_{0}(\ell \vartheta) t_{+}(\vartheta)$

Due to the requirement (43) we can replace $t_{+}$by $t_{-}$and obtain

$$
\begin{aligned}
T_{+}(\vartheta) & =\int_{0}^{\infty} \mathrm{d} \ell \ell J_{0}(\ell \vartheta) \int_{0}^{\infty} \mathrm{d} \theta \theta J_{4}(\ell \theta) T_{-}(\theta) \\
& =\int_{0}^{\infty} \mathrm{d} \theta \theta T_{-}(\theta) G(\vartheta, \theta)
\end{aligned}
$$

where we defined the function

$G(\vartheta, \theta)=\int_{0}^{\infty} \mathrm{d} \ell \ell J_{0}(\ell \vartheta) J_{4}(\ell \theta)$.

In $\mathrm{S} 02$ it was shown that

$G(\vartheta, \theta)=\left(\frac{4}{\theta^{2}}-\frac{12 \vartheta^{2}}{\theta^{4}}\right) H(\theta-\vartheta)+\frac{1}{\theta} \delta_{\mathrm{D}}(\theta-\vartheta)$,

where $H(x)$ is the Heaviside step function and $\delta_{\mathrm{D}}$ the Dirac delta function. Thus,

$T_{+}(\vartheta)=T_{-}(\vartheta)+\int_{\vartheta}^{\infty} \mathrm{d} \theta \theta T_{-}(\theta)\left(\frac{4}{\theta^{2}}-\frac{12 \vartheta^{2}}{\theta^{4}}\right)$

Hence, for any function $T_{-}(\vartheta)$ we can calculate from (47) the corresponding function $T_{+}$so that this pair of functions satisfies (43). In a similar way, $T_{-}$can be obtained from a chosen function $T_{+}$, from

$T_{-}(\vartheta)=T_{+}(\vartheta)+\int_{0}^{\vartheta} \mathrm{d} \theta \theta T_{+}(\theta)\left(\frac{4}{\vartheta^{2}}-\frac{12 \theta^{2}}{\vartheta^{4}}\right)$.

\subsection{General E/B-mode decomposition on a finite interval}

We now return to the original question of this paper, namely to find a separation of E- and B-modes from correlation functions given on a finite interval. Thus we choose a function $T_{-}(\vartheta)$ which is non-zero only on the interval $\vartheta_{\text {min }} \leq \vartheta \leq \vartheta_{\max }$. The upper limit of the integral in (47) can then be replaced by $\vartheta_{\max }$, and we see immediately that $T_{+}(\vartheta)=0$ for $\vartheta>\vartheta_{\max }$. If we require that 
$T_{+}(\vartheta)$ vanishes for $\vartheta<\vartheta_{\min }$, then the following two conditions need to be satisfied:

$\int_{\vartheta_{\min }}^{\vartheta_{\max }} \frac{\mathrm{d} \vartheta}{\vartheta} T_{-}(\vartheta)=0=\int_{\vartheta_{\min }}^{\vartheta_{\max }} \frac{\mathrm{d} \vartheta}{\vartheta^{3}} T_{-}(\vartheta)$.

Thus, if we choose a function $T_{-}$with is non-zero only on a finite interval, the corresponding function $T_{+}$vanishes outside this interval as well, provided $T_{-}$satisfied the two integral constraints (49). The argument which led to the constraints (49) only applies for $\vartheta_{\min }>0$; for $\vartheta_{\text {min }}=0$, these conditions become obsolete.

Conversely, if we choose a function $T_{+}$which vanishes outside the interval $\vartheta_{\min } \leq \vartheta \leq \vartheta_{\max }$, the corresponding function $T_{-}$ is zero outside the same interval if $T_{+}$satisfies the two constraints

$\int_{\vartheta_{\min }}^{\vartheta_{\max }} \mathrm{d} \vartheta \vartheta T_{+}(\vartheta)=0=\int_{\vartheta_{\text {min }}}^{\vartheta_{\max }} \mathrm{d} \vartheta \vartheta^{3} T_{+}(\vartheta)$

if $\vartheta_{\max }<\infty$.

In fact, it is straightforward to show that the definition of $T_{+}$ in terms of $T_{-}$as given by (47) implies the relations (50) provided $\vartheta_{\text {min }}>0$; this latter condition is needed to guarantee the existence of the integrals that occur. Similarly, the definition (48) implies the validity of (49) if $\vartheta_{\max }<\infty$.

We can check these results in a different way, by expanding the Bessel functions into a power series and comparing the result, when inserted into (42) term-by-term. Using

$$
\begin{aligned}
& J_{0}(z)=1-\frac{z^{2}}{4}+\left(\frac{z}{2}\right)^{4} \sum_{k=0}^{\infty} \frac{\left(-z^{2} / 4\right)^{k}}{(k+2) !(k+2) !} \\
& J_{4}(z)=\left(\frac{z}{2}\right)^{4} \sum_{k=0}^{\infty} \frac{\left(-z^{2} / 4\right)^{k}}{k !(k+4) !}
\end{aligned}
$$

we see that the integral (42) of each term is guaranteed to exist if $T_{ \pm}$have finite support, which we assume henceforth. The first two terms of $J_{0}$, when inserted into the first of (42), vanish because of (50). We next compare terms proportional to $\ell^{(2 k+4)}$ of $t_{ \pm}$. For $t_{-}$, this term is

$$
t_{-k}=\int \mathrm{d} \vartheta \vartheta T_{-}(\vartheta) \frac{\left(-\vartheta^{2} / 4\right)^{(k+2)}}{k !(k+4) !},
$$

whereas for $t_{+}$, the corresponding term reads

$$
t_{+k}=\int \mathrm{d} \vartheta \vartheta T_{+}(\vartheta) \frac{\left(-\vartheta^{2} / 4\right)^{(k+2)}}{(k+2) !(k+2) !} .
$$

Inserting now the expression (47) for $T_{+}$, we find

$$
\begin{aligned}
t_{+k}= & \frac{(-4)^{-(k+2)}}{(k+2) !(k+2) !} \int_{0}^{\infty} \mathrm{d} \vartheta \vartheta^{(2 k+5)} \\
& \times\left[T_{-}(\vartheta)+\int_{\vartheta}^{\infty} \mathrm{d} \theta \theta T_{-}(\theta)\left(\frac{4}{\theta^{2}}-\frac{12 \vartheta^{2}}{\theta^{4}}\right)\right] .
\end{aligned}
$$

For the second term, we interchange the order of integration, after which the $\vartheta$-integral can be evaluated. This then leads to

$$
\begin{aligned}
t_{+k}= & \int_{0}^{\infty} \mathrm{d} \vartheta \vartheta \frac{\left(-\vartheta^{2} / 4\right)^{(k+2)}}{(k+2) !(k+2) !} \\
& \times T_{-}(\vartheta)\left[1+\frac{2}{k+3}-\frac{6}{k+4}\right] .
\end{aligned}
$$

Using the definition of the faculty, it is now easy to show that $t_{+k}=t_{-k}$ for all $k$. Hence, for weight functions with finite support, the equivalence (43) can be demonstrated for each order of $\ell$.

It is interesting to consider two special cases of the definitions $E$ and $B$ that have been investigated in the literature. The first case is that of the aperture dispersion, as discussed in S02. The corresponding functions there were also denoted by $T_{ \pm}$,

$$
\begin{aligned}
T_{+}(x) & =\frac{6\left(2-15 x^{2}\right)}{5}\left[1-\frac{2}{\pi} \arcsin \left(\frac{x}{2}\right)\right] \\
& +\frac{x \sqrt{4-x^{2}}}{100 \pi}\left(120+2320 x^{2}-754 x^{4}+132 x^{6}-9 x^{8}\right)
\end{aligned}
$$

for $0 \leq x \leq 2$, and zero otherwise, and

$T_{-}(x)=\frac{192}{35 \pi} x^{3}\left(1-\frac{x^{2}}{4}\right)^{7 / 2} H(2-x)$.

Here, $x=\vartheta / \theta_{0}$, where $\theta_{0}$ denotes the aperture radius. The functions are plotted in S02; they both vanish for $x>2$. If we consider $T_{+}$as given, then $T_{-}$follows from (48); since $T_{+}$satisfies (50), $T_{-}$shares the property of $T_{+}$to vanish for $x>2$. Conversely, consider $T_{-}$as given. Since $T_{-}=0$ for $x>2$ one obtains immediately from (47) that $T_{+}$shares this property. However, $T_{-}$does not satisfy the relations (49).

The second example is that corresponding to the shear dispersion in a circle, where the corresponding weight functions have been denoted as $S_{ \pm}$in S02. They are

$$
\begin{aligned}
& S_{+}(x)=\frac{1}{\pi}\left[4 \arccos \left(\frac{x}{2}\right)-x \sqrt{4-x^{2}}\right] H(2-x), \\
& S_{-}(x)=\frac{x \sqrt{4-x^{2}}\left(6-x^{2}\right)-8\left(3-x^{2}\right) \arcsin (x / 2)}{\pi x^{4}}
\end{aligned}
$$

for $x \leq 2$, and $S_{-}(x)=4\left(x^{2}-3\right) / x^{4}$ for $x>2$. Thus, whereas $S_{+}$is confined to a finite interval $0 \leq x \leq 2, S_{-}$is not. The reason for this is that $S_{+}$does not satisfy (50); in fact, $S_{+}(x)$ is a non-negative function.

The constraints (49) and (50) also explain the qualitative behavior of the functions $Z_{ \pm}$shown in Fig. 2. The two constraints in either case require that the functions $Z_{ \pm}$have two roots in their range of support. As this was the reason for the small amplitude of the resulting shear signal shown in Fig. 4, it becomes clear that this small amplitude is not due to the specific choice of our ring statistics, but is a generic feature of the more general statistic $E$.

\section{Summary and conclusions}

In this paper we have constructed new second-order cosmic shear statistics which can be used to separate E- and B-modes from measurements of the shear correlation function $\xi_{ \pm}$on a finite interval, after noting that the previously considered shear statistics for separating these modes (aperture dispersion and the E-/B-mode shear correlation functions) require the measurement of the shear correlation on arbitrarily small and/or large angular scales. As shown in Kilbinger et al. (2006), the lack of knowledge of the shear correlation functions on very small angluar scales (where the overlapping of isophotes affects the shape measurements of a pair of galaxies) affects the aperture dispersion at an unexpectedly high level. We defined the circle 
and ring statistics, which can be obtained from the shear correlation functions $\xi_{ \pm}(\vartheta)$ over a finite range of angular scales. The starting point for the definition of the new statistics is the fact that the cross-component of the shear, averaged over a circle, vanishes for a pure E-mode shear fields, whereas the tangential shear component, when integrated over a circle, vanishes for a pure B-mode shear. Both of these statistics are obtained by integrating the shear correlation functions, multiplied with properly defined weight functions, over a finite interval in angular separation. By choosing the parameters of the ring and/or circle, the interval on which the shear correlation shall be probed can be adjusted.

To summarize the use of the ring statistics, one can select the angular range $\eta \Psi \leq \vartheta \leq \Psi$ over which the E-/B-mode separation should be probed and on which a measurement of the shear corelation functions is available. For a given value of $\eta$, the functions $Z_{ \pm}$can be calculated, using the explicit equations from the appendix, and the resulting E-/B-mode ring statistics are obtained from (31). By varying the angular range, one can test for the presence of B-modes over multiple scales.

Motivated by the result, we have shown that there exists a very large family of such weight functions which can be used to separate E- and B-modes on the second-order statistics level from measured shear correlations on a finite interval. Hence, there is a large freedom to choose these weight functions, of which the circle and ring statistics are special cases.

The resulting signal of the E- and B-mode shear statistics is small; this was explicitly demonstrated for the ring statistics (see Fig. 4), but as we argued in Sect. 5.2, such a small signal is expected in general and can be traced back to the integral constraints (49) and (50) that the weight function $T_{ \pm}$have to satisfy if they are to probe the shear correlation for E-/B-modes over a finite interval in angular separation. The small signal implies a correspondingly small signal-to-noise for these statistics. In particular, if fairly narrow ranges of $\vartheta_{\text {min }} \leq \vartheta \leq \vartheta_{\max }$ are to be probed, the corresponding shear data must have high statistical accuracy. We therefore expect our results here to be of particular relevance for the upcoming large cosmic shear surveys.

The relatively small signal-to-noise is the prize to pay for a fairly localized E-/B-mode separation. Using data from a small angular range yields of course a lower signal than if one integrates over a broader range, as is done for example with the aperture dispersion. To maximize the signal-to-noise, one might keep the minimum separation $\eta \Psi$ fixed, and then vary $\Psi$. The localized nature of the ring statistics has, however, the advantage that measurements on different angular scales will be correlated only weakly, so that the corresponding covariance matrix will approach diagonal form. In a future publication, we intend to construct function $T_{ \pm}$which "optimize" the signal-to-noise for the detection of B-modes over a finite range of scales.

The ring and circle statistics presented here can be generalized to higher-order cosmic shear statistics as well. The aperture statistics that were defined to quantify third-order shear statistics (Jarvis et al. 2004; Schneider et al. 2005) are plagued with the same problem as the second-order aperture statistics pointed out by Kilbinger et al. (2006). Hence, one could generalize the circle statistics to $\left\langle C\left(\theta_{1}\right) C\left(\theta_{2}\right) C\left(\theta_{3}\right)\right\rangle$ and related expressions (by taking complex conjugates of different factors), project out the E- and B-modes, and express the result in terms of the shear three-point correlation functions. By taking weighted integrals over finite ranges of $\theta_{i}$, the third-order ring statistics can be obtained. In this way, also the third-order shear can be tested for the presence of B-modes.

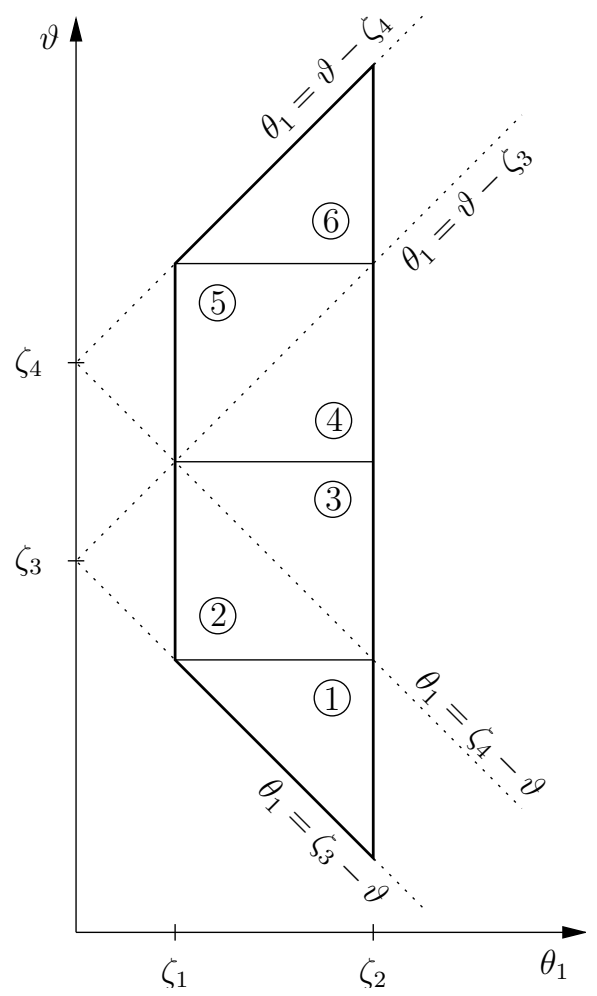

Fig. A.1. The trapezium (solid line) is the integration area in the $\theta_{1}$ $\vartheta$-plane for Eq. (A.1). The devision into six triangular regions is indicated, the numbers correspond to the indices of the integration bounds in (A.3).

Acknowledgements. This work was supported by the Deutsche Forschungsgemeinschaft under the project SCHN 342/6-1 and by the DFG Schwerpunktpgrogramm 1177 "Witnesses of cosmic history".

\section{Appendix A: Explicit calculation of the functions $Z_{ \pm}$}

In this appendix, we carry out the steps that lead to the form of (27), and provide explicit expressions for the function $Z_{-}$. Starting with the final form of (26), then first interchanging the order of integration between $\theta_{2}$ and $\vartheta$, after that changing the order of integration between $\theta_{1}$ and $\vartheta$, we obtain

$$
\begin{aligned}
\langle\mathcal{R R}\rangle= & \int_{\zeta_{3}-\zeta_{2}}^{\zeta_{2}+\zeta_{4}} \frac{\mathrm{d} \vartheta}{\vartheta} \xi_{-}(\vartheta) \int_{\max \left(\zeta_{1}, \vartheta-\zeta_{4}, \zeta_{3}-\vartheta\right)}^{\zeta_{2}} \mathrm{~d} \theta_{1} W_{1}\left(\theta_{1}\right) \\
& \times \int_{\max \left(\zeta_{3}, \vartheta-\theta_{1}\right)}^{\min \left(\zeta_{4}, \vartheta+\theta_{1}\right)} \mathrm{d} \theta_{2} W_{2}\left(\theta_{2}\right) Y_{-}\left(\frac{\vartheta}{\theta_{2}}, \frac{\theta_{1}}{\theta_{2}}\right) .
\end{aligned}
$$

The integration range in the $\theta_{1}-\vartheta$ plane is sketched in Fig. A.1. For this figure, we have chosen the values of $\zeta_{i}$ to satisfy the relations (28); as it becomes clear from this figure, the integral then naturally splits up into six parts. Changing to dimensionless variables by defining

$\vartheta=x \Psi ; \theta_{1}=y_{1} \Psi ; \theta_{2}=y_{2} \Psi$,

with $\Psi=\zeta_{2}+\zeta_{4}$, we can then write

$$
\begin{aligned}
\langle\mathcal{R R}\rangle= & \sum_{i=1}^{6} \int_{a_{i}}^{A_{i}} \frac{\mathrm{d} x}{x} \xi_{-}(x \Psi) \int_{b_{i}}^{B_{i}} \mathrm{~d} y_{1} w_{1}\left(y_{1}\right) \\
& \times \int_{c_{i}}^{C_{i}} \mathrm{~d} y_{2} w_{2}\left(y_{2}\right) Y_{-}\left(\frac{x}{y_{2}}, \frac{y_{1}}{y_{2}}\right)
\end{aligned}
$$


where the various integration bounds are given as

$$
\begin{aligned}
& a_{1}=\eta ; \quad A_{6}=1 ; \quad A_{1}=a_{2}=a_{3}=\frac{3 \eta+1}{4} \\
& a_{4}=a_{5}=A_{2}=A_{3}=\frac{1+\eta}{2} ; \quad a_{6}=A_{4}=A_{5}=\frac{\eta+3}{4} \\
& b_{1}=-b_{4}=-B_{5}=\frac{5 \eta+3}{8}-x \\
& B_{1}=B_{3}=B_{4}=B_{6}=\frac{3(1-\eta)}{8} \\
& b_{2}=b_{5}=\frac{1-\eta}{8} \\
& B_{2}=b_{3}=-b_{6}=\frac{3 \eta+5}{8}-x \\
& c_{1}=c_{2}=c_{3}=c_{4}=\frac{5 \eta+3}{8} ; \quad c_{5}=c_{6}=x-y_{1} \\
& C_{1}=C_{2}=x+y_{1} ; \quad C_{3}=C_{4}=C_{5}=C_{6}=\frac{3 \eta+5}{8},
\end{aligned}
$$

and the functions $w_{i}\left(y_{i}\right)=\Psi W_{i}\left(y_{i} \Psi\right)$. For the choice (30) of the functions $W_{i}$, we obtain

$$
\begin{aligned}
& w_{1}\left(y_{1}\right)=\frac{15\left(8 y_{1}+\eta-1\right)^{2}\left(3-3 \eta-8 y_{1}\right)^{2}}{2(1-\eta)^{5}}, \\
& w_{2}\left(y_{2}\right)=\frac{15\left(8 y_{2}-5 \eta-3\right)^{2}\left(3 \eta+5-8 y_{2}\right)^{2}}{2(1-\eta)^{5}} .
\end{aligned}
$$

The expressions for $\left\langle\mathcal{R R}^{*}\right\rangle$ are identical, except that $\xi_{-}$and $Y_{-}$ are replaced by $\xi_{+}$and $Y_{+}$, respectively. From these explicit expressions, the functions $Z_{ \pm}(x, \eta)$ can be read off readily. They are plotted in Fig. 2.

\section{References}

Bacon, D. J., Refregier, A. R., \& Ellis, R. S. 2000, MNRAS, 318, 625 Bacon, D. J., Massey, R. J., Refregier, A., \& Ellis, R. S. 2003, MNRAS, 344, 673
Bartelmann, M., \& Schneider, P. 2001, Phys. Rep., 340, 291 (BS01)

Catelan, P., Kamionkowski, M., \& Blandford, R. D. 2001, MNRAS, 320, L7

Crittenden, R. G., Natarajan, P., Pen, U.-L., \& Theuns, T. 2001, ApJ, 559, 552

Crittenden, R. G., Natarajan, P., Pen, U.-L., \& Theuns, T. 2002, ApJ, 568, 20 (C02)

Croft, R. A. C., \& Metzler, C. A. 2001, ApJ, 545, 561

Guzik, J., \& Bernstein, G. 2005, PRD, 72, 043503

Heavens, A. F., Refregier, A., \& Heymans, C. E. C. 2000, MNRAS, 319, 649

Heymans, C., \& Heavens, A. 2003, MNRAS, 339, 711

Hoekstra, H., Mellier, Y., van Waerbeke, L., et al. 2005, ApJ, submitted [arXiv: astro-ph/0511089]

Hoekstra, H., Yee, H. K. C., \& Gladders, M. D. 2002, ApJ, 577, 595

Huterer, D., Takada, M., Bernstein, G., \& Jain, B. 2006, MNRAS, 366, 101

Jarvis, M., Bernstein, G. M., Fischer, P., et al. 2003, AJ, 125, 1014

Jarvis, M., Bernstein, G. M., \& Jain, B. 2004, MNRAS, 352, 338

Jarvis, M., Jain, B., Bernstein, G., \& Dolney, D. 2006, ApJ, 644, 71

Jing, Y. P. 2002, MNRAS, 335, L89

Kaiser, N. 1992, ApJ, 388, 272

Kaiser, N. 1995, ApJ, 439, L1

Kaiser, N., Wilson, G., \& Luppino, G. 2000 [arXiv: astro-ph/0003338]

Kilbinger, M., Schneider, P., \& Eifler, T. 2006, A\&A, 457, 19

King, L., \& Schneider, P. 2002, A\&A, 396, 411

King, L., \& Schneider, P. 2003, A\&A, 398, 23

Ma, Z., Hu, W., \& Huterer, D. 2006, ApJ, 636, 21

Maoli, R., van Waerbeke, L., Mellier, Y., et al. 2001, A\&A, 368, 766

Mellier, Y. 1999, ARA\&A, 37, 127

Refregier, A. 2003, ARA\&A, 41, 645

Schneider, P. 1996, MNRAS, 283, 83

Schneider, P. 2003, A\&A, 408, 829

Schneider, P. 2006, in Gravitational Lensing: Strong, Weak \& Micro, Saas-Fee Advanced Course, ed. P. Schneider, C. S. Kochanek, \& J. Wambsganss, 33, (Berlin: Springer-Verlag), 269

Schneider, P., Kilbinger, M., \& Lombardi, M. 2005, A\&A, 431, 9

Schneider, P., van Waerbeke, L., Jain, B., \& Kruse, G. 1998, MNRAS, 296, 873

Schneider, P., van Waerbeke, L., \& Mellier, Y. 2002, A\&A, 389, 729 (S02)

Semboloni, E., Mellier, Y., van Waerbeke, L., et al. 2005, A\&A, submitted (also [arXiv:astro-ph/0511090])

Smith, R. E., Peacock, J. A., Jenkins, A., et al. 2003, MNRAS, 341, 1311

Van Waerbeke, L., Mellier, Y., Erben, T., et al. 2000, A\&A, 358, 30

Van Waerbeke, L., Mellier, Y., Radovich, M., et al. 2001, A\&A, 374, 757

Van Waerbeke, L., Mellier, Y., Pello, R., et al. 2002, A\&A, 393, 369

Van Waerbeke, L., Mellier, Y., \& Hoekstra, H. 2005, A\&A, 429, 75

Wittman, D. M., Tyson, J. A., Kirkman, D., Dell'Antonio, I., \& Bernstein, G. 2000, Nature, 405, 143 